\title{
ON SEPARATELY SUBHARMONIC AND HARMONIC FUNCTIONS
}

\author{
JUHANI RIIHENTAUS \\ Department of Mathematical Sciences, University of Oulu \\ P.O. Box 3000, FI-90014 Oulun yliopisto, Finland \\ riihentaus@member.ams.org \\ and \\ Department of Physics and Mathematics, University of Eastern Finland \\ P.O. Box 111, FI-80101 Joensuu, Finland \\ juhani.riihentaus@uef.fi
}

\begin{abstract}
We improve our previous generalizations to Arsove's and Kołodziej's and Thorbiörnson's results concerning the subharmonicity of a function subharmonic with respect to the first variable and harmonic with respect to the second.
\end{abstract}

Key words: Separately subharmonic, harmonic, quasinearly subharmonic, generalized Laplacian.

AMS 2010 Mathematics Subject Classification: 31C05, 31B25, $31 \mathrm{~B} 05$

\section{INTRODUCTION}

1.1. Solving a long standing problem, Wiegerinck [Wi88, Theorem, p. 770], see also Wiegerinck and Zeinstra [WiZe91, Theorem 1, p. 246], showed that a separately subharmonic function need not be subharmonic. On the other hand, it is an open problem, whether a function which is subharmonic in one variable and harmonic in the other, is subharmonic. For older results on this area, see e.g. Arsove [Ar66, Theorem 2, p. 622], Imomkulov [Im90, Theorem, p. 9], Wiegerinck and Zeinstra [WiZe91, p. 248], Cegrell and Sadullaev [CeSa93, Theorem 3.1, p. 82] and Kołodziej and Thorbiörnson [KoTh96, Theorem 1, p. 463]. The result of Kołodziej and Thorbiörnson includes the results of Arsove, of Cegrell and Sadullaev and of Imomkulov, and reads as follows:

Theorem A Let $\Omega$ be a domain in $\mathbb{R}^{m+n}, m, n \geq 2$. Let $u: \Omega \rightarrow \mathbb{R}$ be such that

(a) for each $y \in \mathbb{R}^{n}$ the function

$$
\Omega(y) \ni x \mapsto u(x, y) \in \mathbb{R}
$$

is subharmonic and $\mathrm{C}^{2}$,

(b) for each $x \in \mathbb{R}^{m}$ the function

is harmonic.

$$
\Omega(x) \ni y \mapsto u(x, y) \in \mathbb{R}
$$

Then $u$ is subharmonic and continuous in $\Omega$.

We improved the result of Kołodziej and Thorbiörnson in a series of papers: [Ri07, , Theorem 3, Theorem 4 and Corollary, pp. 162-164], [Ri072, Theorem 6, p. 234], [Ri073, Theorem 1 and Corollary, pp. 438, 444], [Ri074, Theorem 5.1, Corollary 5.1 and Corollary 5.2, pp. 67-68, 74] and [Ri09, Theorem 4.3.1, Corollary 4.3.3 and Corollary 4.3.4, pp. e2625-e2626]. We will now return to the subject and improve our result still further, see Theorem 2 below.

1.2. However, we begin with improving the above cited results of Arsove and of Cegrell and Sadullaev and our previous generalizations [Ri074, Theorem 4.1, p. 64] and [Ri09, Theorem 4.2.1, p. e2623]. Instead of subharmonic functions (resp. so called quasinearly subharmonic functions n.s.), we will now use quasinearly subharmonic functions. Observe that in certain situations such an approach is indeed useful. One such an example is the following. Armitage and Gardiner [ArGa93, Theorem 1, p. 256] gave a condition which ensures a separately subharmonic function to be subharmonic, and this condition was close to being sharp, see [ArGa93, pp. 255-256]. With the aid of quasinearly subharmonic functions it was, nevertheless, possible to generalize 
and improve their result, see [Ri08, Theorem 4.1 and Corollary 4.5, pp. 8-9, 13] and [Ri09, Theorem 3.3.1 and Corollary 3.3.3, pp. e2621-e2622].

1.3. Our presentation below, including the presented references, is rather detailed. For the notation, and for the definitions and properties of subharmonic functions, nearly subharmonic functions, quasinearly subharmonic functions (and quasinearly subharmonic functions n.s., too) etc., see e.g. [Br69], [He71], [Ta88], [RiTa93], [Ri074], [Ri08], [PaRi08], [Ri09], [PaRi09], [Ri11], and the references therein.

\section{ARSOVE'S RESULT AND ITS IMPROVEMENT}

2.1. Arsove's result is:

Theorem B ([Ar66, Theorem 2, p. 622]) Let $\Omega$ be a domain in $\mathbb{R}^{m+n}, m, n \geq 2$. Let $u: \Omega \rightarrow \mathbb{R}$ be such that

(a) for each $y \in \mathbb{R}^{n}$ the function

$$
\Omega(y) \ni x \mapsto u(x, y) \in \mathbb{R}
$$

is subharmonic,

(b) for each $x \in \mathbb{R}^{m}$ the function

$$
\Omega(x) \ni y \mapsto u(x, y) \in \mathbb{R}
$$

is harmonic,

(c) there is a nonnegative function $\varphi \in \mathcal{L}_{\text {loc }}^{1}(\Omega)$ such that $-\varphi \leq u$.

Then $u$ is subharmonic in $\Omega$.

Arsove's proof was based on mean value operators. Much later Cegrell and Sadullaev [CeSa93, Theorem 3.1, p. 82] gave a new proof using Poisson modification.

2.2. Below in Theorem 1 we generalize the above result of Arsove and of Cegrell and Sadullaev, and also our previous generalizations [Ri074, Theorem 4.1 and Corollary 4.1, pp. 64-65], see also [Ri09, Theorem 4.2.1 and Corollary 4.2.2, p. e2623]. The proof we give below is short and direct.

Theorem 1 Let $\Omega$ be a domain in $\mathbb{R}^{m+n}, m, n \geq 2$, and $K \geq 1$. Let $u: \Omega \rightarrow \mathbb{R}$ be such that

(a) for each $y \in \mathbb{R}^{n}$ the function

$$
\Omega(y) \ni x \mapsto u(x, y) \in \mathbb{R}
$$

is K-quasinearly subharmonic,

(b) for each $x \in \mathbb{R}^{m}$ the function

$$
\Omega(x) \ni y \mapsto u(x, y) \in \mathbb{R}
$$

is harmonic,

(c) there is a nonnegative function $\varphi \in \mathcal{L}_{\text {loc }}^{1}(\Omega)$ such that $-\varphi \leq u$.

Then $u$ is $K$-quasinearly subharmonic in $\Omega$.

Proof. It is easy to see that $u$ is Lebesgue measurable. Therefore also $u_{M}:=\max \{u,-M\}+M, M>0$, is Lebesgue measurable. We must show that $u^{+} \in \mathcal{L}_{\text {loc }}^{1}(\Omega)$ and that each $u_{M}$ satisfies the generalized mean value inequality.

To see that $u^{+} \in \mathcal{L}_{\text {loc }}^{1}(\Omega)$, we proceed as follows. Observe first that $0 \leq u^{+} \leq u_{M} \leq v_{M}:=u+\varphi+M$. To see that $v_{M} \in \mathcal{L}_{\text {loc }}^{1}(\Omega)$ requires only Fubini's Theorem. As a matter of fact, take $\overline{B^{m}(a, R) \times B^{n}(b, R)} \subset \Omega$ arbitrarily. 
Then

$$
\begin{aligned}
0 & \leq \frac{K}{m_{m+n}\left(B^{m}(a, R) \times B^{n}(b, R)\right)} \int_{B^{m}(a, R) \times B^{n}(b, R)} v_{M}(x, y) d m_{m+n}(x, y)= \\
& \leq \frac{K}{m_{m+n}\left(B^{m}(a, R) \times B^{n}(b, R)\right)} \int_{B^{m}(a, R) \times B^{n}(b, R)}[u(x, y)+\varphi(x, y)+M] d m_{m+n}(x, y)= \\
& \leq \frac{K}{v_{m} R^{m}} \int_{B^{m}(a, R)}\left\{\frac{1}{v_{n} R^{n}} \int_{B^{n}(b, R)}[u(x, y)+\varphi(x, y)+M] d m_{n}(y)\right\} d m_{m}(x)= \\
& \leq \frac{K}{v_{m} R^{m}} \int_{B^{m}(a, R)}\left[\frac{1}{v_{n} R^{n}} \int_{B^{n}(b, R)} u(x, y) d m_{n}(y)+\frac{1}{v_{n} R^{n}} \int_{B^{n}(b, R)} \varphi(x, y) d m_{n}(y)+M\right] d m_{m}(x)= \\
& \leq \frac{K}{v_{m} R^{m}} \int_{B^{m}(a, R)}\left[u(x, b)+\frac{1}{v_{n} R^{n}} \int_{B^{n}(b, R)} \varphi(x, y) d m_{n}(y)+M\right] d m_{m}(x)= \\
& \leq \frac{K}{v_{m} R^{m}} \int_{B^{m}(a, R)} u(x, b) d m_{m}(x)+\frac{K}{v_{m} R^{m}} \int_{B^{m}(a, R)}\left[\frac{1}{v_{n} R^{n}} \int_{B^{n}(b, R)} \varphi(x, y) d m_{n}(y)\right] d m_{m}(x)+K M= \\
& \leq \frac{K}{v_{m} R^{m}} \int_{B^{m}(a, R)} u(x, b) d m_{m}(x)+\frac{K}{m_{m+n}\left(B^{m}(a, R) \times B^{n}(b, R)\right)} \int_{B^{m}(a, R) \times B^{n}(b, R)} \varphi(x, y) d m_{m+n}(x, y)+K M \\
& <+\infty .
\end{aligned}
$$

It remains to show that for all $(a, b) \in \Omega$ and $R>0$ such that $\overline{B^{m+n}((a, b), R)} \subset \Omega$,

$$
u_{M}(a, b) \leq \frac{K}{v_{m+n} R^{m+n}} \int_{B^{m+n}((a, b), R)} u_{M}(x, y) d m_{m+n}(x, y) .
$$

To see this, we proceed in the following standard, direct and short way, see e.g. [He71, Proposition 2 c) and proof of Theorem a), pp. 10-11, 32-33] and [Ri074, p. 59]:

$$
\begin{aligned}
& \frac{K}{v_{m+n} R^{m+n}} \int_{B^{m+n}((a, b), R)} u_{M}(x, y) d m_{m+n}(x, y)= \\
& =\frac{v_{m}}{v_{m+n} R^{m+n}} \int_{B^{n}(b, R)}\left[\left(R^{2}-|y-b|^{2}\right)^{\frac{m}{2}} \frac{K}{v_{m}\left(R^{2}-|y-b|^{2}\right)^{\frac{m}{2}}} \int_{B^{m}\left(a, \sqrt{R^{2}-|y-b|^{2}}\right)} u_{M}(x, y) d m_{m}(x)\right] d m_{n}(y) \\
& \geq \frac{v_{m}}{v_{m+n} R^{m+n}} \int_{B^{n}(b, R)}\left(R^{2}-|y-b|^{2}\right)^{\frac{m}{2}} u_{M}(a, y) d m_{n}(y) \geq u_{M}(a, b) .
\end{aligned}
$$

Above we have used, in addition to the fact that, for every $y \in \mathbb{R}^{m}$, the functions $u(\cdot, y)$ are $K$-quasi-nearly subharmonic, also the following lemma. (Observe that the proof of the Lemma, see [He71, proof of Theorem 2 a), p. 15], works also in our slightly more general situation: Recall that in the definition of nearly subharmonic functions, we use instead of the standard condition $v \in \mathcal{L}_{\text {loc }}^{1}(U)$, the slightly weaker condition $u^{+} \in \mathcal{L}_{\text {loc }}^{1}(U)$, see $\left[\mathrm{RiO7}_{4}\right.$, p. 51].)

Lemma. ([He71, Theorem 2 a), p. 15]) Let $v$ be nearly subharmonic (in the generalized sense, defined above) in a domain $U$ of $\mathbb{R}^{N}, N \geq 2, \psi \in \mathcal{L}^{\infty}\left(\mathbb{R}^{N}\right), \psi \geq 0, \psi(x)=0$ when $|x| \geq \alpha$ and $\psi(x)$ depends only on $|x|$. Then $\psi \star v \geq v$ and $\psi \star v$ is subharmonic in $U_{\alpha}$, provided $\int \psi(x) d m_{N}(x)=1$, where $U_{\alpha}=\left\{x \in U: \overline{B^{N}(x, \alpha)} \subset U\right\}$.

\section{AN IMPROVEMENT TO THE RESUlt OF KOŁODZIEJ AND THORBIÖRNSON}

3.1. In our generalization to the cited result of Kołodziej and Thorbiörnson, Theorem A above, we will use the generalized Laplacian, defined with the aid of the Blaschke-Privalov operators, see e.g. [Sa41, p. 451], [Ru50, pp. 278-279], [Sh56, p. 91], [Br69, p. 20], [Sh71, p. 374], [Sh78, p. 29] and [RiTa93, p. 1130]. Let D be a 
domain in $\mathbb{R}^{N}, N \geq 2$, and $f: D \rightarrow \mathbb{R}, f \in \mathcal{L}_{\text {loc }}^{1}(D)$. We write

$$
\begin{aligned}
& \Delta_{*} f(x):=\liminf _{r \rightarrow 0} \frac{2(N+2)}{r^{2}} \cdot\left[\frac{1}{v_{N} r^{N}} \int_{B^{N}(x, r)} f\left(x^{\prime}\right) d m_{N}\left(x^{\prime}\right)-f(x)\right], \\
& \Delta^{*} f(x):=\limsup _{r \rightarrow 0} \frac{2(N+2)}{r^{2}} \cdot\left[\frac{1}{v_{N} r^{N}} \int_{B^{N}(x, r)} f\left(x^{\prime}\right) d m_{N}\left(x^{\prime}\right)-f(x)\right] .
\end{aligned}
$$

If $\Delta_{*} f(x)=\Delta^{*} f(x)$, then write $\Delta f(x):=\Delta_{*} f(x)=\Delta^{*} f(x)$.

If $f \in \mathrm{C}^{2}(D)$, then

$$
\Delta f(x)=\left(\sum_{j=1}^{N} \frac{\partial^{2} f}{\partial x_{j}^{2}}\right)(x)
$$

the standard Laplacian with respect to the variable $x=\left(x_{1}, x_{2}, \ldots, x_{N}\right)$. More generally, if $x \in D$ and $f \in t_{2}^{1}(x)$, i.e. $f$ has an $\mathcal{L}^{1}$ total differential of order 2 at $x$, then $\Delta f(x)$ equals with the pointwise Laplacian of $f$ at $x$, i.e.

$$
\Delta f(x)=\sum_{j=1}^{N} D_{j j} f(x) .
$$

Here $D_{j j} f$ represents a generalization to the usual $\frac{\partial^{2} f}{\partial x_{j}^{2}}, j=1,2, \ldots, N$. See e.g. [CaZy61, p. 172], [Sh56, p. 498], [Sh71, p. 369] and [Sh78, p. 29].

Recall that there exist functions which are not $\mathcal{C}^{2}$ but for which the generalized Laplacian is nevertheless continuous, perhaps in the extended sense (in $([0,+\infty], q)$, where $q$ is the spherical metric), see e.g. [Sh78, p. 31] and [Ri074, Example 5 and Example 6, pp. 66-67] and [Ri09, Example 1 and Example 2, pp. e2624-e2625].

If $f$ is subharmonic on $D$, it follows from [Sa41, p. 451] (see also [Ru50, Lemma 2.2, p. 280]) that $\Delta f(x)=$ $\Delta_{*} f(x)=\Delta^{*} f(x) \in \mathbb{R}$ for almost every $x \in D$.

Below we use the following notation. Let $\Omega$ is a domain in $\mathbb{R}^{m+n}, m, n \geq 2$, and $u: \Omega \rightarrow \mathbb{R}$. If $y \in \mathbb{R}^{n}$ is such that the function

$$
\Omega(y) \ni x \mapsto f(x):=u(x, y) \in \mathbb{R}
$$

is in $\mathcal{L}_{\text {loc }}^{1}(\Omega(y))$, then we write $\Delta_{1 *} u(x, y):=\Delta_{*} f(x), \Delta_{1}^{*} u(x, y):=\Delta^{*} f(x)$, and $\Delta_{1} u(x, y):=\Delta f(x)$.

3.2. Then to our generalization to our previous result [Ri074, Theorem 5.1, pp. 67-68] (or [Ri09, Theorem 4.3.1, p. e2625] (where no proofs are given!)) and thus also to the result of Kołodziej and Thorbiörnson [KoTh96, Theorem 1, p. 463], Theorem A above. Though our proof will follow the main lines of [Ri074, proof of Theorem 5.1, pp. 67-72], it is different enough, nevertheless, to warrant that it be given in complete detail here: Now our assumption (d) is essentially milder than our previous assumptions (d) and (e).

Theorem 2 Let $\Omega$ be a domain in $\mathbb{R}^{m+n}, m, n \geq 2$. Let $u: \Omega \rightarrow \mathbb{R}$ be such that for each $\left(x^{\prime}, y^{\prime}\right) \in \Omega$ there is $\left(x_{0}, y_{0}\right) \in \Omega$ and $r_{1}>0, r_{2}>0$ such that $\left(x^{\prime}, y^{\prime}\right) \in B^{m}\left(x_{0}, r_{1}\right) \times B^{n}\left(y_{0}, r_{2}\right) \subset \overline{B^{m}\left(x_{0}, r_{1}\right) \times B^{n}\left(y_{0}, r_{2}\right)} \subset \Omega$ and such that the following conditions are satisfied:

(a) For each $y \in \overline{B^{n}\left(y_{0}, r_{2}\right)}$ the function

$$
\overline{B^{m}\left(x_{0}, r_{1}\right)} \ni x \mapsto u(x, y) \in \mathbb{R}
$$

is continuous, and subharmonic in $B^{m}\left(x_{0}, r_{1}\right)$.

(b) For each $x \in \overline{B^{m}\left(x_{0}, r_{1}\right)}$ the function

$$
\overline{B^{n}\left(y_{0}, r_{2}\right)} \ni y \mapsto u(x, y) \in \mathbb{R}
$$

is continuous, and harmonic in $B^{n}\left(y_{0}, r_{2}\right)$.

(c) For each $y \in B^{n}\left(y_{0}, r_{2}\right)$ one has $\Delta_{1 *} u(x, y)<+\infty$ for each $x \in B^{m}\left(x_{0}, r_{1}\right)$, possibly with the exception of a polar set in $B^{m}\left(x_{0}, r_{1}\right)$.

(d) There are a set $H \subset B^{n}\left(y_{0}, r_{2}\right)$, dense in $B^{n}\left(y_{0}, r_{2}\right)$, and a set $K \subset B^{m}\left(x_{0}, r_{1}\right)$, dense in $B^{m}\left(x_{0}, r_{1}\right)$, such that

(d1) for each $y \in H$, for almost every $x \in B^{m}\left(x_{0}, r_{1}\right)$ and for each $x \in K$,

$$
\Delta_{1} u\left(x^{\prime}, y\right) \rightarrow \Delta_{1} u(x, y) \in \mathbb{R}
$$

as $x^{\prime} \rightarrow x, x^{\prime} \in K$, and 
(d2) for each $y \in B^{n}\left(y_{0}, r_{2}\right) \backslash H$ and for almost every $x \in B^{m}\left(x_{0}, r_{1}\right)$,

$$
\Delta_{1} u\left(x, y^{\prime}\right) \rightarrow \Delta_{1} u(x, y) \in \mathbb{R}
$$

$$
\text { as } y^{\prime} \rightarrow y, y^{\prime} \in H \text {. }
$$

Then $u$ is subharmonic in $\Omega$.

Proof. Choose $r_{1}^{\prime}, r_{2}^{\prime}$ such that $0<r_{1}^{\prime}<r_{1}, 0<r_{2}^{\prime}<r_{2}$, and such that $\left(x^{\prime}, y^{\prime}\right) \in B^{m}\left(x_{0}, r_{1}^{\prime}\right) \times B^{n}\left(y_{0}, r_{2}^{\prime}\right)$. It is sufficient to show that $u \mid B^{m}\left(x_{0}, r_{1}^{\prime}\right) \times B^{n}\left(y_{0}, r_{2}^{\prime}\right)$ is subharmonic. For the sake of convenience of notation, we change the roles of $r_{j}$ and $r_{j}^{\prime}, j=1,2$. We divide the proof into several steps.

Step 1 Construction of an auxiliar dense set $G$.

For each $k \in \mathbb{N}$ write

$$
A_{k}:=\left\{x \in \overline{B^{m}\left(x_{0}, r_{1}\right)}:-k \leq u(x, y) \leq k \quad \text { for each } \quad y \in \overline{B^{n}\left(y_{0}, r_{2}\right)}\right\} .
$$

Clearly $A_{k}$ is closed, and

$$
\overline{B^{m}\left(x_{0}, r_{1}\right)}=\bigcup_{k=1}^{+\infty} A_{k}
$$

Write

$$
G:=\bigcup_{k=1}^{+\infty} \operatorname{int} A_{k} .
$$

It follows from Baire's theorem that $G$ is dense in $B^{m}\left(x_{0}, r_{1}\right)$.

Step 2 The functions $\Delta_{1 r} u(x, \cdot): B^{n}\left(y_{0}, r_{2}\right) \rightarrow \mathbb{R}$ (see the definition below), $x \in G, 0<r<r_{x}:=\operatorname{dist}\left(x, \overline{B^{m}\left(x_{0}, r_{1}\right)} \backslash\right.$ $G)$, are nonnegative and harmonic.

For each $(x, y) \in B^{m}\left(x_{0}, r_{1}\right) \times B^{n}\left(y_{0}, r_{2}\right)$ and each $r, 0<r<\operatorname{dist}\left(x, \partial B^{m}\left(x_{0}, r_{1}^{\prime}\right)\right)\left(\right.$ observe that $\operatorname{dist}\left(x, \partial B^{m}\left(x_{0}, r_{1}^{\prime}\right)\right)>$ $\left.r_{1}^{\prime}-r_{1}>0\right)$, write

$$
\begin{aligned}
\Delta_{1 r} u(x, y) & :=\frac{2(m+2)}{r^{2}} \cdot\left[\frac{1}{v_{m} r^{m}} \int_{B^{m}(x, r)} u\left(x^{\prime}, y\right) d m_{m}\left(x^{\prime}\right)-u(x, y)\right] \\
& =\frac{2(m+2)}{r^{2}} \cdot \frac{1}{v_{m} r^{m}} \int_{B^{m}(0, r)}\left[u\left(x+x^{\prime}, y\right)-u(x, y)\right] d m_{m}\left(x^{\prime}\right) .
\end{aligned}
$$

Since $u(\cdot, y)$ is subharmonic, $\Delta_{1 r} u(x, y)$ is defined and nonnegative. Suppose then that $x \in G$ and $0<r<r_{x}$. Since $\overline{B^{m}(x, r)} \subset G$ and $A_{k} \subset A_{k+1}$ for all $k=1,2, \ldots, \overline{B^{m}(x, r)} \subset$ int $A_{N}$ for some $N \in \mathbb{N}$. Therefore

$$
-N \leq u\left(x^{\prime}, y\right) \leq N \quad \text { for all } \quad x^{\prime} \in B^{m}(x, r) \text { and } y \in B^{n}\left(y_{0}, r_{2}\right)
$$

and hence

$$
-2 N \leq u\left(x+x^{\prime}, y\right)-u(x, y) \leq 2 N \quad \text { for all } \quad x^{\prime} \in B^{m}(0, r) \quad \text { and } \quad y \in B^{n}\left(y_{0}, r_{2}\right) .
$$

To show that $\Delta_{1 r} u(x, \cdot)$ is continuous, pick an arbitrary sequence $y_{j} \rightarrow \tilde{y}_{0}, y_{j}, \tilde{y}_{0} \in B^{n}\left(y_{0}, r_{2}\right), j=1,2, \ldots$ Using then (1), Lebesgue Dominated Convergence Theorem and the continuity of $u(x, \cdot)$, one sees easily that $\Delta_{1 r} u(x, \cdot)$ is continuous.

To show that $\Delta_{1 r} u(x, \cdot)$ satisfies the mean value equality, take $\tilde{y}_{0} \in B^{n}\left(y_{0}, r_{2}\right)$ and $\rho>0$ arbitrarily such that $\overline{B^{n}\left(\tilde{y}_{0}, \rho\right)} \subset B^{n}\left(y_{0}, r_{2}\right)$. Because of (1) we can use Fubini's Theorem. Thus

$$
\begin{aligned}
\frac{1}{v_{n} \rho^{n}} \int_{B^{n}\left(\tilde{y}_{0}, \rho\right)} \Delta_{1 r} u(x, y) d m_{n}(y) & =\frac{1}{v_{n} \rho^{n}} \int_{B^{n}\left(\tilde{y}_{0}, \rho\right)}\left\{\frac{2(m+2)}{r^{2}} \cdot \frac{1}{v_{m} r^{m}} \int_{B^{m}(0, r)}\left[u\left(x+x^{\prime}, y\right)-u(x, y)\right] d m_{m}\left(x^{\prime}\right)\right\} d m_{n}(y) \\
& =\frac{2(m+2)}{r^{2}} \cdot \frac{1}{v_{m} r^{m}} \int_{B^{m}(0, r)}\left\{\frac{1}{v_{n} \rho^{n}} \int_{B^{n}\left(\tilde{y}_{0}, \rho\right)}\left[u\left(x+x^{\prime}, y\right)-u(x, y)\right] d m_{n}(y)\right\} d m_{m}\left(x^{\prime}\right) \\
& =\frac{2(m+2)}{r^{2}} \cdot \frac{1}{v_{m} r^{m}} \int_{B^{m}(0, r)}\left[u\left(x+x^{\prime}, \tilde{y}_{0}\right)-u\left(x, \tilde{y}_{0}\right)\right] d m_{m}\left(x^{\prime}\right) \\
& =\Delta_{1 r} u\left(x, \tilde{y}_{0}\right) .
\end{aligned}
$$


Step 3 The functions $\Delta_{1} u(x, \cdot): B^{n}\left(y_{0}, r_{2}\right) \rightarrow \mathbb{R}, x \in G \cap A$, are defined, nonnegative and harmonic. Here

$$
A:=\bigcap_{k=1}^{+\infty} A\left(y_{k}\right)
$$

where $H=\left\{y_{k}, k=1,2, \ldots\right\}$ (we may clearly suppose that $H$ is countable), and, for arbitrary $y \in B^{n}\left(y_{0}, r_{2}\right)$,

$$
A(y):=\left\{x \in B^{m}\left(x_{0}, r_{1}\right): \Delta_{1 *} u(x, y)=\Delta_{1}^{*} u(x, y)=\Delta_{1} u(x, y) \in \mathbb{R}\right\} .
$$

By [Ru50, Lemma 2.2, p. 280] (see also [Sa41, p. 451] and [Sh71, p. 376]), $m_{m}\left(B^{m}\left(x_{0}, r_{1}\right) \backslash A(y)\right)=0$ for each $y \in B^{n}\left(y_{0}, r_{2}\right)$.

Take $x \in G \cap A$ and a sequence $r_{j} \rightarrow 0,0<r_{j}<r_{x}, j=1,2, \ldots$, arbitrarily. By [He71, Corollary 3 a), p. 6] (or [AG01, Lemma 1.5.6, p. 16]) we see that the family

$$
\Delta_{1 r_{j}} u(x, \cdot): B^{n}\left(y_{0}, r_{2}\right) \rightarrow \mathbb{R}, j=1,2, \ldots,
$$

of nonnegative and harmonic functions is either uniformly equicontinuous and locally uniformly bounded, or else

$$
\sup _{j=1,2, \ldots} \Delta_{1 r_{j}} u(x, \cdot) \equiv+\infty .
$$

On the other hand, since $x \in G \cap A$, we know that for each $y_{k} \in H, k=1,2, \ldots$,

$$
\Delta_{1 r_{j}} u\left(x, y_{k}\right) \rightarrow \Delta_{1} u\left(x, y_{k}\right) \in \mathbb{R}
$$

as $j \rightarrow+\infty$. Therefore, by [Vä71, Theorem 20.3, p. 68] and by [He71, c), p. 2] (or [ArGa01, Theorem 1.5.8, p. 17]), the limit

$$
\Delta_{1} u(x, \cdot)=\lim _{j \rightarrow+\infty} \Delta_{1 r_{j}} u(x, \cdot)
$$

exists and defines a harmonic function in $B^{n}\left(y_{0}, r_{2}\right)$. Since the limit is clearly independent of the considered sequence $r_{j}$, the claim follows.

Step 4 The function $\Delta_{1} u(\cdot, \cdot) \mid(G \cap K \cap A \cap B) \times B^{n}\left(y_{0}, r_{2}\right)$ has a continuous extension $\tilde{\Delta}_{1} u(\cdot, \cdot):(A \cap B) \times$ $B^{n}\left(y_{0}, r_{2}\right) \rightarrow \mathbb{R}$. Moreover, the functions $\tilde{\Delta}_{1} u(x, \cdot): B^{n}\left(y_{0}, r_{2}\right) \rightarrow \mathbb{R}, x \in A \cap B$, are nonnegative and harmonic. Here

$$
B:=\bigcap_{k=1}^{+\infty} B\left(y_{k}\right),
$$

where, for arbitrary $y \in B^{n}\left(y_{0}, r_{2}\right)$, we use the notation

$$
B(y):=\left\{x \in B^{m}\left(x_{0}, r_{1}\right): \Delta_{1} u\left(x^{\prime}, y\right) \rightarrow \Delta_{1} u(x, y) \text { as } x^{\prime} \rightarrow x, x^{\prime} \in K\right\} .
$$

Using the assumption (d1), one sees easily that $G \cap K \cap A \cap B$ is dense in $A \cap B$.

To show the existence of the desired continuous extension, it is clearly sufficient to show that for each $\left(\tilde{x}_{0}, \tilde{y}_{0}\right) \in(A \cap B) \times B^{n}\left(y_{0}, r_{2}\right)$, the limit

$$
\lim _{(x, y) \rightarrow\left(\tilde{x}_{0}, \tilde{y}_{0}\right),(x, y) \in(G \cap K \cap A \cap B) \times B^{n}\left(y_{0}, r_{2}\right)} \Delta_{1} u(x, y)
$$

exists. (This is of course standard, see e.g. [Di60, (3.15.5), p. 54].) To see this, it is sufficient to show that, for an arbitrary sequence $\left(x_{j}, y_{j}\right) \rightarrow\left(\tilde{x}_{0}, \tilde{y}_{0}\right),\left(x_{j}, y_{j}\right) \in(G \cap K \cap A \cap B) \times B^{n}\left(y_{0}, r_{2}\right), j=1,2, \ldots$, the limit

$$
\lim _{j \rightarrow+\infty} \Delta_{1} u\left(x_{j}, y_{j}\right)
$$

exists.

That this limit indeed exists, is seen as above, just using the facts:

- the functions $\Delta_{1} u\left(x_{j}, \cdot\right), j=1,2, \ldots$, are nonnegative and harmonic in $B^{n}\left(y_{0}, r_{2}\right)$, by Step 3;

- for each $y_{k} \in H, k=1,2, \ldots, \Delta_{1} u\left(x_{j}, y_{k}\right) \rightarrow \Delta_{1} u\left(\tilde{x}_{0}, y_{k}\right) \in \mathbb{R}$ as $j \rightarrow+\infty$.

(See again [He71, Corollary 3 a), p. 6] (or [AG01, Lemma 1.5.6, p. 16]) and [Vä71, Theorem 20.3, p. 68]). That the functions $\tilde{\Delta}_{1} u(x, \cdot): B^{n}\left(y_{0}, r_{2}\right) \rightarrow \mathbb{R}, x \in A \cap B$, are harmonic, see [He71, c), p. 2] (or [ArGa01, Theorem 1.5.8, p. 17])).

Step 5 For each $x \in B^{m}\left(x_{0}, r_{1}\right)$ the functions

$$
B^{n}\left(y_{0}, r_{2}\right) \ni y \mapsto \tilde{v}(x, y):=\int G_{6}^{G_{B^{m}\left(x_{0}, r_{1}\right)}(x, z) \tilde{\Delta}_{1} u(z, y) d m_{m}(z) \in \mathbb{R}}
$$


and

$$
B^{n}\left(y_{0}, r_{2}\right) \ni y \mapsto \tilde{h}(x, y):=u(x, y)+\tilde{v}(x, y) \in \mathbb{R}
$$

are harmonic. Above and below $G_{B^{m}\left(x_{0}, r_{1}\right)}(x, z)$ is the Green function of the ball $B^{m}\left(x_{0}, r_{1}\right)$, with $x$ as a pole.

Using Fubini's Theorem one sees easily that for each $x \in B^{m}\left(x_{0}, r_{1}\right)$ the function $\tilde{v}(x, \cdot)$ satisfies the mean value equality. To see that $\tilde{v}(x, \cdot)$ is harmonic, it is sufficient to show that $\tilde{v}(x, \cdot) \in \mathcal{L}_{\text {loc }}^{1}\left(B^{n}\left(y_{0}, r_{2}\right)\right)$. Using just Fatou's Lemma, one sees that $\tilde{v}(x, \cdot)$ is lower semicontinuous, hence superharmonic. Therefore either $\tilde{v}(x, \cdot) \equiv$ $+\infty$ or else $\tilde{v}(x, \cdot) \in \mathcal{L}_{\text {loc }}^{1}\left(B^{n}\left(y_{0}, r_{2}\right)\right)$. The following argument shows that the former alternative cannot occur. Indeed, for each $x \in A \cap B$ and for each $y_{k} \in H, k=1,2, \ldots$, we see, using the definition of the (continuous) function $\tilde{\Delta}_{1} u(\cdot, \cdot)$ and $(\mathrm{d} 1)$, that

$$
\tilde{\Delta}_{1} u\left(x, y_{k}\right)=\lim _{x^{\prime} \rightarrow x, x^{\prime} \in G \cap K \cap A \cap B} \tilde{\Delta}_{1} u\left(x^{\prime}, y_{k}\right)=\lim _{x^{\prime} \rightarrow x, x^{\prime} \in G \cap K \cap A \cap B} \Delta_{1} u\left(x^{\prime}, y_{k}\right)=\Delta_{1} u\left(x, y_{k}\right) \in \mathbb{R} .
$$

Hence $\tilde{v}\left(x, y_{k}\right)=v\left(x, y_{k}\right) \in \mathbb{R}$ for each $x \in B^{m}\left(x_{0}, r_{1}\right)$ and $y_{k} \in H, k=1,2, \ldots$ (See (3) in Step 6 below for the definition of $v(\cdot, \cdot): B^{m}\left(x_{0}, r_{1}\right) \times B^{n}\left(y_{0}, r_{2}\right) \rightarrow \mathbb{R}$.) Therefore, for each $x \in B^{m}\left(x_{0}, r_{1}\right)$, the function $\tilde{v}(x, \cdot)$ and thus also the function $\tilde{h}(x, \cdot)=u(x, \cdot)+\tilde{v}(x, \cdot)$ are harmonic.

Step 6 For each $y \in B^{n}\left(y_{0}, r_{2}\right)$ the function

$$
B^{m}\left(x_{0}, r_{1}\right) \ni x \mapsto \tilde{h}(x, y) \in \mathbb{R}
$$

is harmonic.

With the aid of the version of Riesz's Decomposition Theorem, given in [Ru50, 1.3. Theorem II, p. 279, and p. 278, too] (see also [Sh56, Theorem 1, p. 499]), for each $y \in B^{n}\left(y_{0}, r_{2}\right)$ one can write

$$
u(x, y)=h(x, y)-v(x, y),
$$

where

$$
v(x, y):=\int G_{B^{m}\left(x_{0}, r_{1}\right)}(x, z) \Delta_{1} u(z, y) d m_{m}(z)
$$

and $h(\cdot, y)$ is the least harmonic majorant of $u(\cdot, y) \mid B^{m}\left(x_{0}, r_{1}\right)$. Here $v(\cdot, y)$ is continuous and superharmonic in $B^{m}\left(x_{0}, r_{1}\right)$.

As shown above in $(2), v\left(\cdot, y_{k}\right)=\tilde{v}\left(\cdot, y_{k}\right)$ for each $y_{k} \in H, k=1,2, \ldots$ Therefore $\tilde{h}\left(\cdot, y_{k}\right)=h\left(\cdot, y_{k}\right)$, and thus $\tilde{h}\left(\cdot, y_{k}\right)$ is harmonic for each $y_{k} \in H, k=1,2, \ldots$

To see that $\tilde{h}(\cdot, y)$ is harmonic also for $y \in B^{n}\left(y_{0}, r_{2}\right) \backslash H$, take $\tilde{y}_{0} \in B^{n}\left(y_{0}, r_{2}\right) \backslash H$ arbitrarily, and proceed in the following way. Take $z \in A \cap B \cap A\left(\tilde{y}_{0}\right) \cap C\left(\tilde{y}_{0}\right)$ arbitrarily, where, for arbitrary $y \in B^{n}\left(y_{0}, r_{2}\right) \backslash H$,

$$
C(y):=\left\{z \in B^{m}\left(x_{0}, r_{1}\right): \Delta_{1 *} u\left(z, y^{\prime}\right) \rightarrow \Delta_{1 *} u(z, y) \text { as } y^{\prime} \rightarrow y, y^{\prime} \in H\right\} .
$$

Since $z \in A\left(\tilde{y}_{0}\right)$, we have $\Delta_{1 *} u\left(z, \tilde{y}_{0}\right)=\Delta_{1} u\left(z, \tilde{y}_{0}\right) \in \mathbb{R}$. Thus we may also suppose that $\Delta_{1 *} u\left(z, y^{\prime}\right)=\Delta_{1} u\left(z, y^{\prime}\right) \in$ $\mathbb{R}$. Using then our assumption (d2) and the continuity of $\tilde{\Delta}_{1} u(\cdot, \cdot)$, we see that

$$
\Delta_{1} u\left(z, \tilde{y}_{0}\right)=\tilde{\Delta}_{1} u\left(z, \tilde{y}_{0}\right)
$$

for every $z \in A \cap B \cap A\left(\tilde{y}_{0}\right) \cap C\left(\tilde{y}_{0}\right)$. Therefore, $\tilde{v}\left(x, \tilde{y}_{0}\right)=v\left(x, \tilde{y}_{0}\right)$ and thus $\tilde{h}\left(x, \tilde{y}_{0}\right)=h\left(x, \tilde{y}_{0}\right)$ for each $x \in$ $B^{m}\left(x_{0}, r_{1}\right)$.

Step 7 The use of the results of Lelong and of Avanissian.

By Steps 5 and 6 we know that $\tilde{h}(\cdot, \cdot)=h(\cdot, \cdot)$ is separately harmonic in $B^{m}\left(x_{0}, r_{1}\right) \times B^{n}\left(y_{0}, r_{2}\right)$. By Lelong's result [Le61, p. 561] (see also [Av67, Théorème 1, pp. 4-5]) $\tilde{h}(\cdot, \cdot)$ is harmonic and thus locally bounded above in $B^{m}\left(x_{0}, r_{1}\right) \times B^{n}\left(y_{0}, r_{2}\right)$. Therefore also $u(\cdot, \cdot)$ is locally bounded above in $B^{m}\left(x_{0}, r_{1}\right) \times B^{n}\left(y_{0}, r_{2}\right)$. But then it follows from [Av61, Théorème 9, p. 140] (see also [Le45, Théorème 1 bis, p. 315], [Ar66, Theorem 1, p. 622], [Le69, Proposition 3, p. 24], [Ri89,Theorem 1, p. 69], [ArGa93, Theorem 1, p. 256] and [Ri08, Corollary 4.5, p. 13]) that $u(\cdot, \cdot)$ is subharmonic on $B^{m}\left(x_{0}, r_{1}\right) \times B^{n}\left(y_{0}, r_{2}\right)$.

Remark 1 Observe that the assumption (d2) was needed only to see that

$$
\Delta_{1} u(x, y)=\tilde{\Delta}_{1} u(x, y) \text { for almost every } x \in B^{m}\left(x_{0}, r_{1}\right) \text { and for each } y \in B^{n}\left(y_{0}, r_{2}\right) \backslash H .
$$

(At this point one might recall that the functions $\tilde{\Delta}_{1} u(x, \cdot): B^{n}\left(y_{0}, r_{2}\right) \rightarrow \mathbb{R}, x \in B^{m}\left(x_{0}, r_{1}\right)$, are harmonic.) From the above proof one sees easily that the assumption (d), that is (d1) and (d2), can be replaced by: 
$\left(d^{*}\right)$ For every $y \in B^{n}\left(y_{0}, r_{2}\right)$, for almost every $x \in B^{m}\left(x_{0}, r_{1}\right)$ and for each $x \in K$,

$$
\Delta_{1} u\left(x^{\prime}, y\right) \rightarrow \Delta_{1} u(x, y) \in \mathbb{R}
$$

as $x^{\prime} \rightarrow x, x^{\prime} \in K$

Though our Theorem 2 might still be considered somewhat technical, it has, nevertheless, the following concise corollaries, both of which already improve the result of Kołodziej and J. Thorbiörnson.

Corollary 1 ([Ri074, Corollary 5.1, p. 74] and [Ri09, Corollary 4.3.3, p. e2626]) Let $\Omega$ be a domain in $\mathbb{R}^{m+n}$, $m, n \geq 2$. Let $u: \Omega \rightarrow \mathbb{R}$ be such that

(a) for each $y \in \mathbb{R}^{n}$ the function

$$
\Omega(y) \ni x \mapsto u(x, y) \in \mathbb{R}
$$

is continuous and subharmonic,

(b) for each $x \in \mathbb{R}^{m}$ the function

$$
\Omega(x) \ni y \mapsto u(x, y) \in \mathbb{R}
$$

is harmonic,

(c) for each $y \in \mathbb{R}^{n}$ the function

$$
\Omega(y) \ni x \mapsto \Delta_{1} u(x, y) \in[0,+\infty]
$$

is defined, continuous (with respect to the spherical metric), and finite for all $x$, except at most of a polar set $E(y)$ in $\Omega(y)$.

Then $u$ is subharmonic in $\Omega$.

Corollary 2 ([Ri073, Corollary, p. 444]) Let $\Omega$ be a domain in $\mathbb{R}^{m+n}, m, n \geq 2$. Let $u: \Omega \rightarrow \mathbb{R}$ be such that

(a) for each $y \in \mathbb{R}^{n}$ the function

$$
\Omega(y) \ni x \mapsto u(x, y) \in \mathbb{R}
$$

is continuous and subharmonic,

(b) for each $x \in \mathbb{R}^{m}$ the function

$$
\Omega(x) \ni y \mapsto u(x, y) \in \mathbb{R}
$$

is harmonic,

(c) for each $y \in \mathbb{R}^{n}$ the function

$$
\Omega(y) \ni x \mapsto \Delta_{1} u(x, y) \in \mathbb{R}
$$

is defined and continuous.

Then $u$ is subharmonic in $\Omega$.

\section{References}

[ArGa93] D.H. Armitage and S.J. Gardiner, Conditions for separately subharmonic functions to be subharmonic, Potential Anal., 2 (1993), 255-261.

[ArGa01] D.H. Armitage and S.J. Gardiner, Classical Potential Theory, Springer-Verlag, London, 2001.

[Ar66] M.G. Arsove, On subharmonicity of doubly subharmonic functions, Proc. Amer. Math. Soc., 17 (1966), 622-626.

[Av61] V. Avanissian, Fonctions plurisousharmoniques et fonctions doublement sousharmoniques, Ann. Sci. École Norm. Sup., 78 (1961), 101-161.

[Av67] V. Avanissian, Sur l'harmonicité des fonctions séparément harmoniques, in: Séminaire de Probabilités (Univ. Strasbourg, Février 1967), 1 (1966/1967), pp. 101-161, Springer, Berlin, 1967.

[Br69] M. Brelot, Éléments de la Théorie Classique du Potentiel, Centre de Documentation Universitaire, Paris, 1969 (Third Edition).

[CaZy61] A.P. Calderon and A. Zygmund, Local properties of solutions of elliptic partial differential equations, Studia Math., 20 (1961), 171-225.

[CeSa93] U. Cegrell and A. Sadullaev, Separately subharmonic functions, Uzbek. Math. J., 1 (1993), 78-83.

[Di60] J. Dieudonné, Foundations of Modern Analysis, Academic Press, New York, 1960.

[He71] M. Hervé, Analytic and Plurisubharmonic Functions in Finite and Infinite Dimensional Spaces, Lecture Notes in Mathematics, Vol. 198, Springer, Berlin · Heidelberg · New York, 1971.

[Im90] S.A. Imomkulov, Separately subharmonic functions (in Russian), Dokl. USSR, 2 (1990), 8-10.

[KoTh96] S. Kołodziej and J. Thorbiörnson, Separately harmonic and subharmonic functions, Potential Anal., 5 (1996), 463-466. 
[Le45] P. Lelong, Les fonctions plurisousharmoniques, Ann. Sci. École Norm. Sup., 62 (1945), 301-338.

[Le61] P. Lelong, Fonctions plurisousharmoniques et fonctions analytiques de variables réelles, Ann. Inst. Fourier, Grenoble, 11 (1961), 515-562.

[Le69] P. Lelong, Plurisubharmonic Functions and Positive Differential Forms, Gordon and Breach, London, 1969.

[PaRi08] M. Pavlović and J. Riihentaus, Classes of quasi-nearly subharmonic functions, Potential Anal. 29 (2008), 89-104.

[PaRi09] M. Pavlović and J. Riihentaus, Quasi-nearly subharmonic functions in locally uniformly homogeneous spaces, Positivity, 15, no. 1 (2009), 1-10.

[Ri89] J. Riihentaus, On a theorem of Avanissian-Arsove, Expo. Math., 7 (1989), 69-72.

[Ri07 $]$ J. Riihentaus, Separately quasi-nearly subharmonic functions, in: Complex Analysis and Potential Theory, Proceedings of the Conference Satellite to ICM 2006, Tahir Aliyev Azeroğlu, Promarz M. Tamrazov (eds.), Gebze Institute of Technology, Gebze, Turkey, September 8-14, 2006, World Scientific, Singapore, 2007, pp. 156-165.

[Ri072] J. Riihentaus, On the subharmonicity of separately subharmonic functions, in: Proceedings of the 11 th WSEAS International Conference on Applied Mathematics (MATH'07), Dallas, Texas, USA, March 22-24, 2007, Kleanthis Psarris, Andrew D. Jones (eds.), WSEAS, 2007, pp. 230-236.

[Ri07, J. Riihentaus, On separately harmonic and subharmonic functions, Int. J. Pure Appl. Math., 35, no. 4 (2007), 435-446.

[Ri074] J. Riihentaus, Subharmonic functions, generalizations and separately subharmonic functions, The XIV-th Conference on Analytic Functions, July 22-28, 2007, Chełm, Poland, in: Scientific Bulletin of Chełm, Section of Mathematics and Computer Science, 2 (2007), 49-76.

[Ri08] J. Riihentaus, Quasi-nearly subharmonicity and separately quasi-nearly subharmonic functions, J. Inequal. Appl., 2008, Article ID 149712, 15 pages, 2008.

[Ri09] J. Riihentaus, Subharmonic functions, generalizations and separately subharmonic functions: A survey, 5th World Congress of Nonlinear Analysts (WCNA '08), July 2 - 9, 2008, Orlando, Florida, USA, in: Nonlinear Analysis, 71 (2009), e2613-e2627.

[Ri11] J. Riihentaus, Domination conditions for families of quasinearly subharmonic functions, International Journal of Mathematics and Mathematical Sciences/New Trends in Geometric Function Theory 2011, 2011 (2011), Article ID 729849, 9 pages.

[RiTa93] J. Riihentaus and P.M. Tamrazov, On subharmonic extension and the extension in the Hardy-Orlicz classes (English and Ukrainian summaries), Ukrain. Mat. Zh., 45, no. 8 (1993), 1260-1271.

[Ru50] W. Rudin, Integral representation of continuous functions, Trans. Amer. Math. Soc., 68 (1950), 278-286.

[Sa41] S. Saks, On the operators of Blaschke and Privaloff for subharmonic functions, Rec. Math. (Mat. Sbornik), 9 (51) (1941), 451-456.

[Sh56] V.L. Shapiro, Generalized laplacians, Amer. J. Math., 78 (1956), 497-508.

[Sh71] V.L. Shapiro, Removable sets for pointwise subharmonic functions, Trans. Amer. Math. Soc., 159 (1971), 369-380.

[Sh78] V.L. Shapiro, Subharmonic functions and Hausdorff measure, J. Diff. Eq., 27 (1978), $28-45$.

[Ta88] P.M. Tamrazov, Removal of singularities of subharmonic, plurisubharmonic functions and their generalizations (English and Ukrainian summaries), Ukrain. Mat. Zh., 40, no. 6 (1988), 683-694 (Russian); translation in Ukrainian Math. J., 40, no. 6 (1988), 573-582.

[Vä71] J. Väisälä, Lectures on n-Dimensional Quasiconformal Mappings, Lecture Notes in Mathematics, Vol. 229, Springer, Berlin · Heidelberg · New York, 1971.

[Wi88] J. Wiegerinck, Separately subharmonic functions need not be subharmonic, Proc. Amer. Math. Soc., 104 (1988), 770-771.

[WiZe91] J. Wiegerinck and R. Zeinstra, Separately subharmonic functions: when are they subharmonic, in: Proceedings of Symposia in Pure Mathematics, vol. 52, part 1, Eric Bedford, John P. D'Angelo, Robert E. Greene, Steven G. Krantz (eds.), Amer. Math. Soc., Providence, Rhode Island, 1991, pp. 245-249. 